\title{
ADAM-15 Disintegrin-Like Domain Structure and Function
}

\section{Dong Lu ${ }^{1,2}$, Mike Scully ${ }^{1}$, Vijay Kakkar ${ }^{1}$ and Xinjie Lu ${ }^{1, *}$}

1 Thrombosis Research Institute, Manresa Road, London, SW3 6LR, UK;

E-Mails: d13@sanger.ac.uk (D.L.); mscully@tri-london.ac.uk (M.S.); president@tri-kakkar.ch (V.K.)

2 Sanger Institute, Wellcome Trust Genome Campus, Hinxton, Cambridge, CB10 1SA, UK

* Author to whom correspondence should be addressed; E-Mail: xlu@tri-london.ac.uk; Tel.: +44-0207-351-8312; Fax: +44-0207-351-8324.

Received: 30 August 2010; in revised form: 13 October 2010 / Accepted: 18 October 2010 / Published: 19 October 2010

\begin{abstract}
The ADAM (a disintegrin-like and metalloproteinase) proteins are a family of transmembrane cell-surface proteins with important functions in adhesion and proteolytic processing in all animals. Human ADAM-15 is the only member of the ADAM family with the integrin binding motif Arg-Gly-Asp (RGD) in its disintegrin-like domain. This motif is also found in most snake venom disintegrins and other disintegrin-like proteins. This unique RGD motif within ADAM-15 serves as an integrin ligand binding site, through which it plays a pivotal role in interacting with integrin receptors, a large family of heterodimeric transmembrane glycoproteins. This manuscript will present a review of the RGD-containing disintegrin-like domain structures and the structural features responsible for their activity as antagonists of integrin function in relation to the canonical RGD template.
\end{abstract}

Key words: ADAM protein; Snake venom toxin; disintegrin; integrin; RGD-motif

\section{Introduction}

The "disintegrin" terminology was initially applied in 1990 to describe a family of cysteine-rich, RGD-containing proteins, isolated from the venom of snakes that inhibit platelet aggregation and integrin-mediated cell adhesion [1-3]. Subsequently, homologous proteins in which the arginine residue was replaced within the RGD motif including the motifs: KGD [4,5], MGD [6], VGD [7], 
WGD or MLDG [8,9] were also adopted into the disintegrin family. The RGD sequence is also found in proteins such as decorsin [10] and ornatin [11] from leech toxins, and variabilin [12] from hard tick toxin. The term "disintegrins" was eventually reserved for a particular form of snake venom toxins, and the term "disintegrin-like protein" for RGD proteins with similar properties but different general structures, including the disintegrin-like/cysteine-rich $(\mathrm{D} / \mathrm{C})$ domains of the PIII class snake venom metalloproteinases (SVMP) [13,14]; the ADAM (a disintegrin-like and metalloproteinase) [15-18] and ADAMTS (ADAM with thrombospondin motifs) [19,20]; ADAMTSL (ADAMTS-like) families [21] and MDC (metalloproteinase disintegrin-like cysteine-rich) proteins [22,23]. The primary sequences of disintegrin-like domains in the ADAMs family were homologous to those found in snake venom disintegrins. These proteins constitute one subfamily of the so-called adamalysins, which is a protein family belonging to metzincin superfamily of metalloproteinases. Members of this large and conserved protein family have been isolated from a variety of organisms, including mammals, reptiles and invertebrates. Of the 34 ADAM proteins described including the 19 human ADAMs, human ADAM-15 (also called MDC-15, ADAM metallopeptidase domain 15 or metargidin) is the only ADAM protein with the RGD integrin ligand consensus motif in a position analogous to that found in snake venom disintegrins. The RGD sequence is followed by an additional cysteine residue that is not present in RGD-type snake venom disintegrins and has only been detected in non-RGD-type SVMP and ADAM proteins. In this review, we focus on the disintegrin-like domain in ADAM 15 and its structure and function.

\section{Overview of the ADAM-15 Gene Structure}

Human ADAM-15 was discovered in a screen for novel ADAMs by PCR [24]. Expression of a disintegrin-like protein had already been observed in cultured human vascular cells and in vivo [25]. ADAM-15 was named metargidin since it carried an RGD sequence in a similar position as snake venom disintegrins (metalloproteinase-RGD-disintegrin protein) [26].

Human ADAM 15 is located at 1q21.3 of chromosome 1, the largest human chromosome, with $\sim 8 \%$ of all human genetic information starting at 153,290,386 bp and ending at 153,301,876 bp from the pter (phosphotriesterase related) and reported to have six transcripts and 11,491 bases. Large introns (intron 1, $1183 \mathrm{bp}$ ) occur on the $5^{\prime}$ and $3^{\prime}$ sides of the gene with a cluster of exons between them (Figure 1) [27].

Figure 1. Schematic of partial chromosome 1 with ADAM-15 gene indicated by an arrow.

\section{ADAM-15 gene}

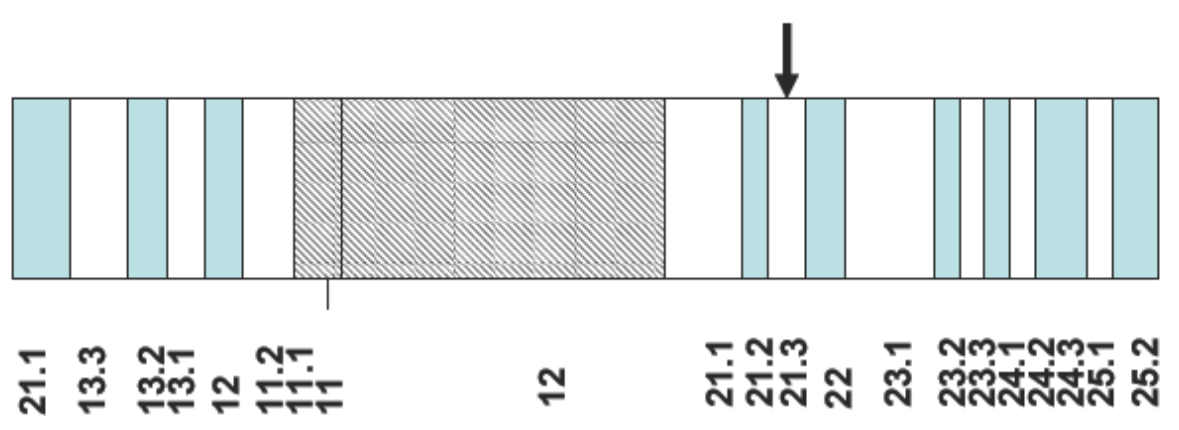


The gene for ADAM-15 contains 23 exons varying in size from 63-316 bp and 22 introns ranging between 79-1283 bp [28]. The ADAM-15 protein isoforms deduced have combinations of cytosolic regulatory protein interacting motifs with one or both of the almost identical proline-rich regions encoded by exons 20 and 21, where the residues RxLPxxP are indispensable for nephrocystin SH3 binding [29].

Human ADAM-15 contains a signal peptide sequence (1 to 17 amino acids (aa)) (Figure 2), followed by a pro-peptide or pro-domain (18-206 aa) thought to function as an intramolecular chaperone (IMC). The pro-domain is cleaved from the metalloproteinase domain by furin [30], a membrane associated endoprotease that cleaves precursor proteins on the C-terminal side of the consensus sequence.

Figure 2. Domain structures of ADAMs compared to snake venom metalloproteinases (SVMP). Members of the ADAM gene family are classified as membrane-anchored ADAMs containing cysteine-rich domain, cytosolic tail, disintegrin-like domain, epidermal growth factor-like domain, metalloproteinase domain, Pro-peptide domain and transmembrane (TM) domain. SVMP can be classified into four subgroups ((P-I to P-IV). S.P. denotes signal peptide.

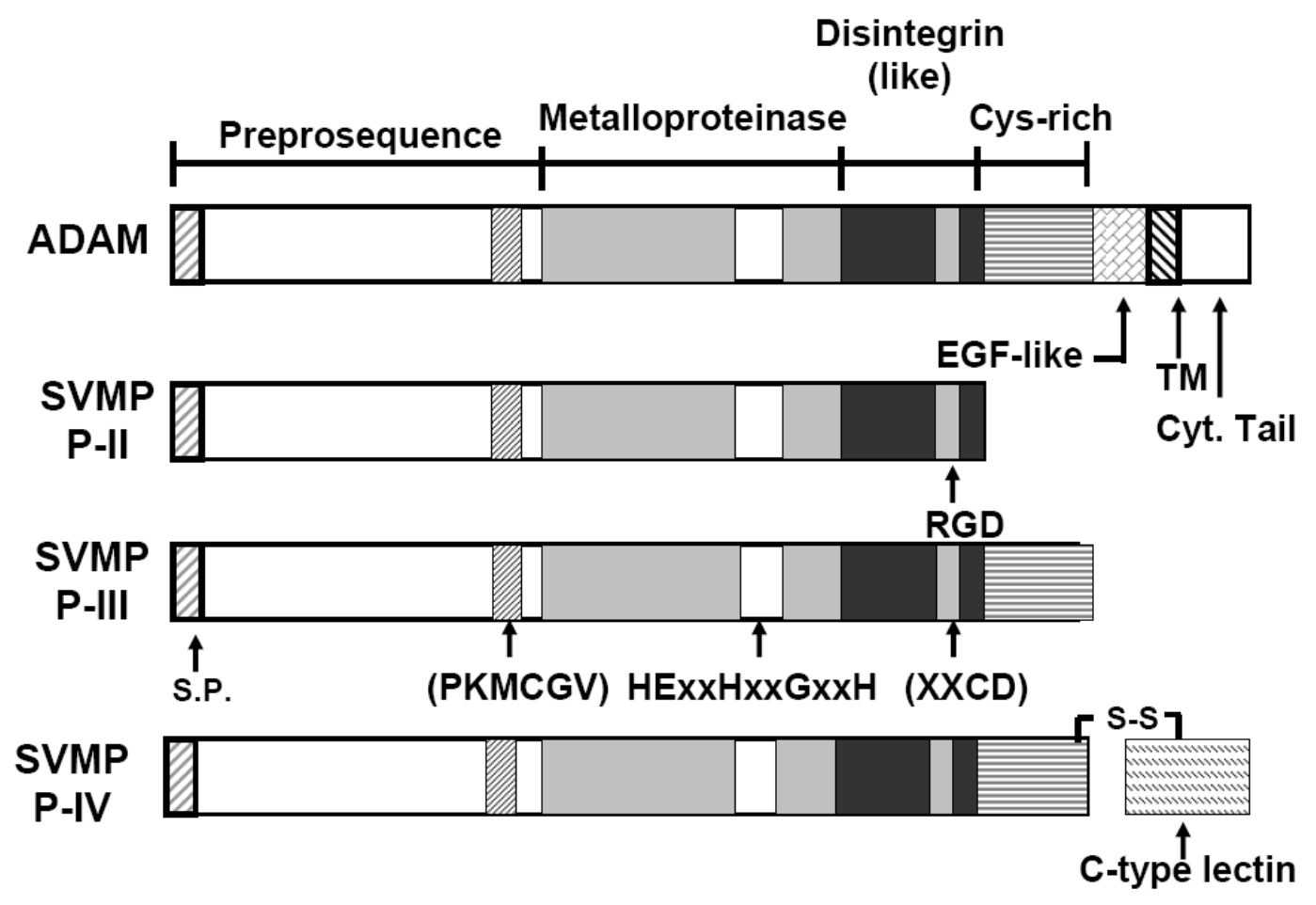

\section{Overview of the Structural Domains of ADAM 15}

The pro-domain maintains the metalloproteinase site of ADAM in an inactive state through a cysteine switch [31] similar to that of matrix metalloproteinases (MMPs) and other reprolysins. In this regard, ADAMs, including ADAM-15, are reprolysin-like proteins. The MMPs or matrixins are synthesized as zymogens, which in the case of soluble matrixins are secreted while other members of the family remain bound to the cell surface. The cysteine switch motif in ADAMs may play a role during ADAM biosynthesis. The cysteine residue preferentially coordinates the active site zinc atom 
sequestering the metalloproteinase domain in an inactive conformation. There are several zinc interacting sites in ADAM-15 (179, 348, 352, 358 aa). Inhibitors of the early secretory pathway block the processing of ADAM-15 and ADAM-9, thus positioning the location of ADAMs processing and activation at the trans-Golgi network [32]. The secondary function of the pro-domain is to chaperone proper folding of the ADAMs, especially the metalloproteinase domain since an ADAM-10 construct lacking the pro-domain is catalytically inactive in vivo [33].

The metalloproteinase domain of ADAM-15 (207-419 aa) and other ADAMs are well conserved, but only 25 out of 40 ADAMs, including ADAM-15 (348-359 aa) and ADAMs 1, 8-10, 12, 13, 16, 17 , 19-21, 24-26, 28, 30, 33-40, have the zinc binding catalytic site consensus sequence HExxHxxGxxHD where $x$ is any amino acid. Three His residues and a water molecule tetrahedrally coordinate the zinc, and the Glu residue acts as a catalytic base [34].

The disintegrin-like domain is downstream of the metalloproteinase domain. The ADAM-15 disintegrin-like domain contains 90 aa $\left(\mathrm{Met}^{420}\right.$ to $\mathrm{Glu}^{510}$ ), while in other ADAMs this domain contains 60-90 aa. ADAM-15 and has 15 Cys residues showing sequence similarity to the snake venom disintegrins [35].

The cysteine-rich domain of ADAM-15 (511-656 aa) is thought to regulate cell fusion and may be involved in the activation of latent ADAM-15 and removal of the pro-domain through mechanisms that are not fully elucidated $[17,36]$.

An EGF-like domain (657-685 aa) is downstream of the cysteine-rich domain, named for its similarity to epidermal growth factor (EGF) and other related growth factors and containing six, highly conserved cysteine residues with characteristic spacing [37]. Certain data suggest the EGF-like domain is involved in substrate specificity including substrate cleavage and recognition [38].

ADAM-15 and many others are type I membrane proteins anchored to the surface of the cell through the extracellular domain (207-696 aa), TM domain (697-717 aa) including a putative phosphorylation site (715 aa) and cytoplasmic domain near the C-terminus (718-814 aa). The cytoplasmic domain of ADAM-15 interacts with endophilin I and the sorting nexin 9. In contrast, all the ADAMTSs lack a TM domain and are secreted proteases.

The cytosolic portion (cytoplasmic tail) of ADAM-15 (718-814 aa) and many other ADAMs vary in length (between 40-250 aa) and sequence composition. Similar to other proteolytically active ADAMs, the cytosolic part of ADAM-15 is rich in proline-rich consensus binding sites motif (766-772 aa and 801-806 aa). The cytosolic domain of ADAM-15 is encoded by exons used alternatively in normal tissues giving rise to splice variants with different compositions of putative protein binding motifs [29]. Certain ADAM-15 variants have been associated with poor survival of breast cancer patients [39].

ADAM-15 has another putative phosphorylation site (tyrosine 735 aa) as do many other ADAMs for serine-threonine and/or tyrosine kinases. Phosphorylation of ADAMs may serve to modulate adaptor functions of the protein to assemble complexes of proteins at sites of functional activity.

\section{Integrin Interactions of the Disintegrin-Like Domain of ADAM-15}

The initial identification of disintegrin-like domains within mammalian ADAMs led to the hypothesis that these regions interact with integrins similar to the related domains in snake venom 
proteins [17]. There is now considerable evidence that the extracellular domains of ADAMs interact with integrins. Recombinant disintegrin-like domains have been identified with a consensus-binding motif, CRxxxxxCDxxExC, in their disintegrin loops [40]. These interactions influence cell adhesion and cell-cell interactions including those dependent upon the integrins: $\alpha_{2} \beta_{1}, \alpha_{\mathrm{IIb}} \beta_{3}, \alpha_{4} \beta_{1}, \alpha_{4} \beta_{7}, \alpha_{5} \beta_{1}$, $\alpha_{6} \beta_{1}, \alpha_{6} \beta_{4}, \alpha_{9} \beta_{1}, \alpha_{V} \beta_{3}$ and $\alpha_{V} \beta 5[41,42]$.

\section{Structural Model of the Disintegrin-Like Domains of ADAMs Proteins}

The disintegrin-like domain (D-domain), which is located downstream of the metalloprotease domain, consists of 60 to 90 aa with 6 to 15 Cys residues. Most D-domains of ADAMs have an XCD motif with the exception of ADAM-15, which contains the RGD sequence (484-486) [43] similar to the snake venom disintegrins. Snake venom disintegrins are known to be potent inhibitors of various integrins. Snake venom disintegrins usually have a RGD motif that confers the ability to interact with integrins [44]. The disulfide bridge of RGD-containing disintegrins has been evaluated by chemical methods, NMR spectroscopy and crystallography. The most striking feature is the consistency of the disulfide bonds around the RGD sequence leading to the proposal of an "RGD-containing loop" in each protein, which may be important to their potency and selectivity. NMR studies of this loop in snake venom proteins, including kistrin [45-47], flavoridin [48], echistatin [49-51], albolabrin [52] and dendroaspin [53] along with the crystal structure of trimestatin [54], show that the RGD sequence is presented at the apex of a $\beta$-turn. Although the active sequence in most disintegrins is the RGD tripeptide, some members of the family contain other sequences such as KGD, MVD, MLD, VGD, ECD, or MDG (single letter amino acid code) in complimentary positions and have been characterized as integrin-binding motifs [55]. The disintegrin-like domains of ADAMs and the P-III group SVMPs is larger than the RGD-disintegrins, and most of them have an XCD motif (where $\mathrm{X}$ is any amino acid) in their disintegrin-like domains, with the exception of ADAM-15 which contains the RGD sequence [56]. P-III group SVMPs comprising the metalloproteinase, disintegrin-like and cysteine-rich domains belong to the ADAM/adamalysin/reprolysin family [14,57-61]. Several ADAMs share a sequence $\mathrm{Rx}_{6} \mathrm{DLPE}$ in the D-domain, which can bind avidly to $\alpha_{9} \beta_{1}$ e.g., ADAM-1, $-2,-12$, and -15 , whereas ADAM-10 and -17 do not since they lack this motif [56].

In the study of metalloproteinase domain-containing proteins including the active sequence in terms of structure and function, the crystal structure of the entire ectodomain of mature ADAM-22 [62] reported following the crystallographic studies of two PIII SVMP proteins, VAP1 (vascular apoptosisinducing protein-1) and VAP2B (vascular apoptosis-inducing protein 2B), proved very useful $[63,64]$. Overlaying the SVMP structures on ADAM-22 revealed a positional shift in the D-domain and C-domains (the cysteine-rich domain, 530-676 aa) in ADAM-22 relative to the corresponding domains in the SVMPs.

The D-domain that follows the metalloproteinase domain (M-domain) is seen in VAP1 and VAP2B and divided into 2 sub-domains, the "D-shoulder" (Ds) and "D-arm" (Da) (Figure 3). Both the Ds (residues 396-440) and Da subdomains (residues 441-487) contain calcium-binding sites [64-66]. ADAM-22 contains three putative calcium ions, two in the D-domain and one in the M-domain M, metalloproteinase-like domain (residues 233-435). The Ds- and Da subdomains consist of a series of turns and two short regions of antiparallel $\beta$-sheet forming a continuous $\mathrm{C}$-shaped structure, which, 
along with the $\mathrm{N}$-terminal region of the $\mathrm{C}$-domain, forms a "C $\mathrm{C}$-wrist" $(\mathrm{Cw})$ segment. The $\mathrm{Cw}$ segment is followed by a "C-hand" (Ch) segment with a hypervariable region (HVR) at its distal portion [65]. These structural features are summarized in the schematic shown in Figure 3. There are three disulfide bonds in the Ds-segment, three in the Da-segment and one in the $\mathrm{Cw}$-segment, and the segments are connected by single disulfide bonds (Figure 3). X-ray studies of atragin, a protein of P-III family of SVMPs, showed one disulfide bond connecting Ds and Da and another disulfide bond connecting Da and $\mathrm{Cw}$ comprised of one cystine residue in the disintegrin-like loop (XXCD) that caused it to become inaccessible for integrin-binding as in VAP1 and VAP2 [61].

Figure 3. Schematic presentations of the MDC domain. (A) and (B) present orthogonal views of the MDC domain of catrocollastatin/VAP2B. The M-domain, linker, Ds, Da, Cw and $\mathrm{Ch}$ segments, $\mathrm{Zn}^{2+}$ binding site, and the HVR are shown in yellow, gray, cyan, pink, gray, light green, red and blue, respectively. The GM6001 (an inhibitor) bound to the protein molecule is shown in ball and stick representation and three $\mathrm{Ca}$ binding sites are indicated as I-III, adapted with permission [66].
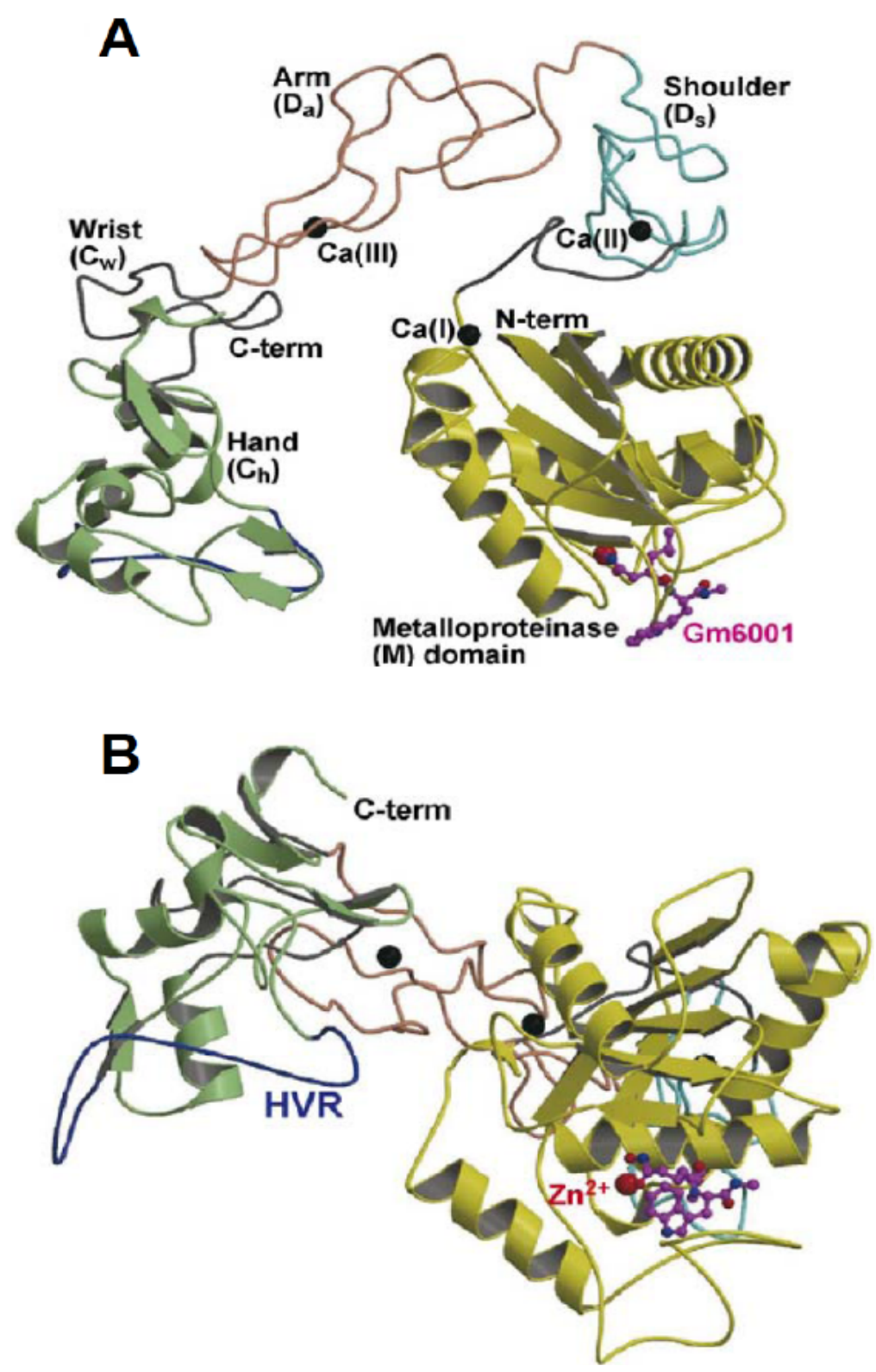


\section{Disintegrin-Like Domain of ADAM-15: Structure and Function}

ADAM-15 has been implicated in cell-cell, cell-matrix interactions and in the proteolysis of molecules on the cell surface or the extracellular matrix [67-70]. The function of ADAM-15 in cell-cell adhesion has been attributed to the D-domain as integrin ligand [71]. Human ADAM-15 provided interesting insights into analyzing the structure/function of the RGD motif dependent interaction with integrin $\alpha_{V} \beta_{3}$ compared to its RGD-independent association with $\alpha_{9} \beta_{1}$ [59]. The study mapped the $\alpha_{9} \beta_{1}$-interaction site to a motif RxxxxxxDLPEF (481-492 aa in human ADAM-15 wherein the RGD motif is at 484-486 aa), that is conserved in all ADAMs excepting ADAM-10 and -17 [59].

\section{Investigation of the Integrin Interaction Using a Recombinant Disintegrin Domain from} ADAM-15 (ddADAM-15) and Various Mutants

The integrin, $\alpha_{9} \beta_{1}$, is widely expressed on smooth muscle and epithelial cells, and mediates adhesion to the extracellular matrix proteins, osteopontin and tenascin-C [72]. We have studied a number of mutants of ddADAM-15 (Figure 4). Recombinant GST-ddADAM-15 and its mutants supported the adhesion of $\alpha_{9} \beta_{1}$-transfected $\mathrm{CHO}$ cells, which were shown to reach $50 \%$ of the maximum number of adherent cells as dd(den)-ADAM-15 > ddADAM-15 > dd(2)-ADAM-15 > dd(12)-ADAM-15 > dd(19)-ADAM-15 > dd(A64)-ADAM-15 [73]. RGD-independent binding of integrin $\alpha_{9} \beta_{1}$ to ddADAM-15 mediates cell-cell interactions [71].

Figure 4. Mutants of disintegrin-like domain of ADAM-15. Sequence alignment of ddADAM-15 and its mutants plotted using CLC protein workbench version 5.2. Numbering is based on the amino acid sequence of ddADAM-15. The dd(A64)-ADAM-15 shows that the $\mathrm{R}$ residue in $\mathrm{R}^{64} \mathrm{GD}$ of ddADAM-15 was replaced by Alanine; $\operatorname{dd}(12)-A D A M-15$ denotes that the disintegrin-like RGD-loop of ADAM-15 was replaced by that of ADAM-12. A similar designation was applied to others. dd(den)-ADAM-15 denotes that the disintegrin-like RGD-loop of ADAM-15 was replaced by that of dendroaspin (den), a disintegrin-like protein [53].

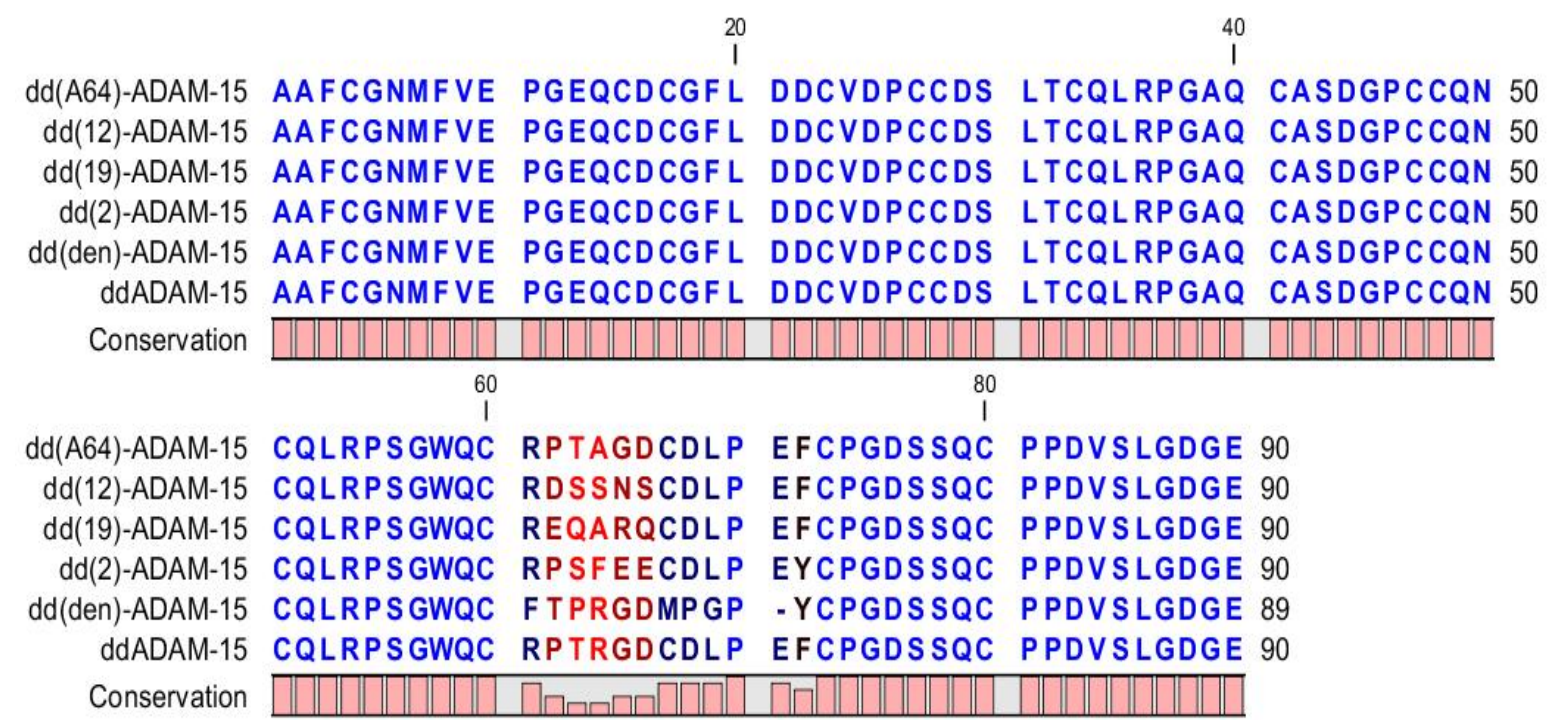


Inhibition of adhesion of $\alpha_{V} \beta_{3}$ integrin-mediated A375-SM cells (a highly metastatic variant of A375 cells, containing $\alpha_{v} \beta_{3}$ integrin) to fibrinogen, by dd-ADAM-15 and its mutants, is ranked as dd(den)-ADAM-15 > dd(2)-ADAM-15 > ddADAM-15 > dd(12)-ADAM-15 > dd(19)-ADAM-15 > dd(A64)-ADAM-15 [73]. Charrier et al. have reported that overexpression of ADAM-15, containing a mutation in the RGD motif in Caco2-BBE (human intestinal cell line) monolayers, decreases Jurkat cell adhesion, and showing ADAM-15-mediated binding of T cells on intestinal epithelial cells is RGD sequence-dependent [74]. This suggests that the $\alpha_{V} \beta_{3}$ and $\alpha_{5} \beta_{1}$ integrins expressed on $\mathrm{T}$ lymphocyte membranes are putative binding partners for epithelial ADAM-15. In vitro experiments confirmed that ddADAM-15 interacts with $\alpha_{V} \beta_{3}$ and $\alpha_{5} \beta_{1}$ integrins on hematopoietic cells [74], and ADAM-15 interaction with these integrins is RGD-dependent [75]. Mosnier et al. reported that ADAM-15 is upregulated in epi- and endothelial cells in close contact with $\alpha_{5} \beta_{1}$-expressing leukocytes, suggesting a role in leukocyte migration [76]. ADAM-15 interacts with $\alpha_{v} \beta_{3}$ and $\alpha_{5} \beta_{1}$ integrins, both of which are involved in endothelial cell migration indicating a possible role in atherosclerosis [77,78].

ddADAM-15 is reported to bind via $\alpha_{2} \beta_{1}$ to HT1080 (a human fibrosarcoma cell line), but the binding is weaker than with dd(den)-ADAM-15, which showed the highest binding ability for HT1080 cells [73). The order of binding potency for dd recombinant proteins to $\alpha_{4} \beta_{1}$-mediated MOLT 4 (human acute lymphoblastic leukemia cell line containing $\alpha_{4} \beta_{1}$ ) cell is demonstrated to be dd(2)-ADAM-15 > dd(19)-ADAM-15 > dd(den)-ADAM-15 = ddADAM-15 > dd(12)-ADAM-15 while dd(A64)-ddADAM-15 showed little/no ability to support cell adhesion compared to its wild-type counterpart. These results suggest that the RGD tripeptide motif may play a role in this binding as dd(A64)-ddADAM-15 failed to bind to this cell line. However, since both dd(den)-ADAM-15 and ddADAM-15 contain the RGD-motif and others do not have the RGD, the difference in potencies emphasizes the importance of the flanking residues in determining potency [73].

Although no inhibition to platelet aggregation was found for dd(2)-ADAM-15, dd(A64)-ADAM-15, dd(19)-ADAM-15 and dd(12)-ADAM-15 with ADP-induced platelet aggregation in platelet-rich plasma, ddADAM-15 showed low activity with a maximum $25 \%$ inhibition at $10 \mu \mathrm{M}$ despite the presence of the RGD motif, which is known to be favored for binding to platelet $\alpha_{\text {IIB }} \beta_{3}$ [73]. Further, yeast-expressed ddADAM-15 inhibited binding of $\alpha_{\text {IIB }} \beta_{3}$ to its biological ligands fibrinogen in a dose-dependent manner. Mutation of the three residues proximal to the RGD tripeptide sequence, RPTRGD sequence to NWKRGD (named NWK mutant), increased its affinity for $\alpha_{\text {IIB }} \beta_{3}$. The NWK mutant had a greater inhibitory action on human platelet aggregation than ddADAM-15 [79], suggesting that flanking amino acid residues are important for activity of the RGD motif.

We have shown that ddADAM-15 can bind to airway smooth muscle cells (ASMCs) and this binding can be modulated by putative disintegrin-like loops within the ddADAM-15 scaffold [80]. This adhesion was mediated by the $\beta_{1}$-asociated integrins including $\alpha_{4} \beta_{1}, \alpha_{5} \beta_{1}, \alpha_{9} \beta_{1}$. Hence, ddADAM-15 can serve as a $\beta_{1}$ integrin antagonist as seen by the inhibition of ASMC binding to fibrinogen. ddADAM-15 inhibited PDGF-induced cell migration with the RGD-motif playing a crucial role as shown by the replacement of the putative disintegrin-like loop with those of ADAM-2, -12 and -19 . We established that fibrinogen, rather than fibronectin, binding was blocked by ddADAM-15 in a dose-dependent manner in $\beta_{1}$-mediated cell binding, implying that ddADAM-15 and fibrinogen share a similar $\beta_{1}$ integrin binding site. Such a region may not be involved in fibronectin binding despite the location of an RGD sequence in the tenth type III repeat of fibronectin, which is the major 
binding site for $\beta_{1}$ integrin with $\alpha_{5} \beta_{1}[81,82]$. The role of RGD in ddADAM-15 may be limited, as this is the only ADAM family protein containing this sequence. It cannot be ruled out that regions beyond the disintegrin-like loop also play a role in integrin-binding since ddADAM-15 and ddADAM-12, which lacks the RGD-motif can interact with $\beta_{1}$-associated $\alpha_{9}$ integrin [68], and -associated $\alpha 5$ integrin [67] in other cell types.

\section{ADAM-15 Is Associated with Diseases}

The role of ADAM-15 in diseases appears to involve mechanisms as diverse as cell-cell interactions, cell-extracellular matrix (ECM) interactions and shedding activity. There is growing evidence of links between ADAM-15 and human diseases including cancer and atherosclerosis. It was reported that mRNA and/or protein levels of ADAM-15 are upregulated in multiple adenocarcinomas including cancer of the breast, stomach, lung, pancreas and prostate [83]. Horiuchi et al. reported that a deficiency of ADAM-15 in a mouse model for retinopathy resulted in reduced neovascularization [84]. Consistently, smaller tumors were formed in the ADAM-15-deficient mice after injection with melanoma cells [85]. Yamada et al. demonstrated that pancreatic cancer cells expressed significantly higher levels ADAM-15 mRNA than normal pancreatic epithelial cells [85]. Najy et al. [86] found that downregulation of ADAM-15 in the prostate cancer cell line, PC3 decreased migration and adhesion to specific extracellular matrix proteins. Using breast cancer cell lines, the same authors reported that ADAM-15 cleaved cadherin E after growth factor deprivation [87]. The cleaved cadherin E bound and transactivated HER2/HER3, resulting in increased migration and proliferation. Thus, enhanced HER2/HER3 signaling is a potential mechanism by which ADAM-15 could contribute to cancer progression. Sun et al. recently reported that ADAM-15 regulates endothelial permeability, which is considered as one of the key cellular processes in the development of inflammatory disorders, including atherosclerosis [88,89], diabetic complications [90] and inflammatory bowel disease [76]. In addition to RGD motif which has an ability to disturb integrin-mediated attachment on the cell surface, the RGD peptides are incorporated into cytoplasm and induce apoptosis [91]. Collectively, several RGD-containing proteins from venom toxins induced apoptosis, such as contortrostatin [92], rhodostomin [93] and salmosin [94]. Since these RGD peptides and RGD-containing proteins interact with integrins, the integrins may serve as targets for anti-cancer agents designed using RGD as a template. Several studies have shown the potential for these RGD proteins to function as integrin antagonists as well as antiagiogenic, antimetastatic and antithrombotic compounds leading to drug development for therapeutic usage [95-97].

\section{Concluding Remarks}

The ddADAM-15 selectively modulates integrin-mediated cell adhesion and ASMC migration. The amino acid sequence in the putative disintegrin-like loop plays a crucial role in controlling the selectivity and specificity of the ADAM proteins in their interaction with particular integrins. The RGD-tripeptide in the putative disintegrin-like loop in ADAM-15 serves as an integrin recognition sequence since conversion of RGD into AGD reduced potency, inhibiting A375-SM cell adhesion to fibrinogen mediated by $\alpha_{V} \beta_{3}$, and showed little/no activity inhibiting $\alpha_{4} \beta_{1}$-mediated MOLT 4 cell attachment. 
Details of conformational changes in the RGD-tri-peptide within ddADAM-15 while interacting with integrins remain unclear. A putative binding model has been constructed based on the 3D structure of integrin $\alpha_{v} \beta_{3}$ in complex, with a cyclic penta-peptide presenting the RGD sequence [98], where the RGD motif of ddADAM-15 is located at $64-66$ aa $\left(\mathrm{R}^{64} \mathrm{GD}^{66}\right)$ and fits a crevice between the propeller ( $\alpha$ subunit) and $\beta$ A ( $\beta_{1}$ subunit) domains on the $\beta_{1}$-associated complex headpiece. Conversely, the RGD motif in atragin (538-540 aa according to the sequence number of atragin), located at the end of the $\eta 4$ helix of the K-like domain, is inaccessible for integrin molecules implying that $\mathrm{K}$-like domains bind to the integrin through a non-RGD region, such as the hypervariable region (HVR) $[61,99]$. In this review, we have mainly described the RGD-containing D-domain in ADAM-15 interacting with integrins, the non-RGD-containing D-domains in other ADAMs are also reported to associate with integrins, e.g., ADAM-23 can bind to $\alpha_{V} \beta_{3}$ [100] and ADAM-28 can interact with $\alpha_{4} \beta_{1}$ [101]. However, the structural basis for these associations has yet to be defined. Therefore, further studies are required to analyze ddADAM-15, ADAM-15 and other ADAMs by X-ray crystallography to gain structural information and increase understanding of ADAM-integrin interaction.

\section{Acknowledgement}

This work was supported by the Thrombosis Research Trust/Garry Weston Foundation. We thank Usha Narayan for editorial assistance.

\section{References}

1. Gould, R.J.; Polokoff, M.A.; Friedman, P.A.; Huang, T.F.; Holt, J.C.; Cook, J.J.; Niewiarowski, S. Disintegrins: a family of integrin inhibitory proteins from viper venoms. Proc. Soc. Exp. Biol. Med. 1990, 195, 168-171.

2. Niewiarowski, S.; McLane, M.A.; Kloczewiak, M.; Stewart, G.J. Disintegrins and other naturally occurring antagonists of platelet fibrinogen receptors. Semin. Hematol. 1994, 31, 289-300.

3. McLane, M.A.; Sanchez, E.E.; Wong, A.; Paquette-Straub, C.; Perez, J.C. Disintegrins. Curr. Drug Targets Cardiovasc. Haematol. Disord. 2004, 4, 327-355.

4. Scarborough, R.M.; Rose, J.W.; Hsu, M.A.; Phillips, D.R.; Fried, V.A.; Campbell, A.M.; Nannizzi, L.; Charo, I.F. Barbourin. A GPIIb-IIIa-specific integrin antagonist from the venom of Sistrurus m. barbouri. J. Biol. Chem. 1991, 266, 9359-9362.

5. Oshikawa, K.; Terada, S. Ussuristatin 2, a novel KGD-bearing disintegrin from Agkistrodon ussuriensis venom. J. Biochem. 1999, 125, 31-35.

6. Nikai, T.; Taniguchi, K.; Komori, Y.; Masuda, K.; Fox, J.W.; Sugihara, H. Primary structure and functional characterization of bilitoxin-1, a novel dimeric P-II snake venom metalloproteinase from Agkistrodon bilineatus venom. Arch. Biochem. Biophys. 2000, 378, 6-15.

7. Calvete, J.J.; Fox, J.W.; Agelan, A.; Niewiarowski, S.; Marcinkiewicz, C. The presence of the WGD motif in CC8 heterodimeric disintegrin increases its inhibitory effect on alphalI(b)beta 3, alpha (v)beta3, and alpha5beta1 integrins. Biochemistry 2002, 41, 2014-2021. 
8. Siigur, E.; Aaspollu, A.; Tu, A.T.; Siigur, J. cDNA cloning and deduced amino acid sequence of fibrinolytic enzyme (lebetase) from Vipera lebetina snake venom. Biochem. Biophys. Res. Commun. 1996, 224, 229-236.

9. Marcinkiewicz, C.; Calvete, J.J.; Marcinkiewicz, M.M.; Raida, M.; Vijay-Kumar, S.; Huang, Z.; Lobb, R.R.; Niewiarowski, S. EC3, a novel heterodimeric disintegrin from Echis carinatus venom, inhibits alpha4 and alpha5 integrins in an RGD-independent manner. J. Biol. Chem. 1999, 274, 12468-12473.

10. Seymour, J.L.; Henzel, W.J.; Nevins, B.; Stults, J.T.; Lazarus, R.A. Decorsin. A potent glycoprotein IIb-IIIa antagonist and platelet aggregation inhibitor from the leech Macrobdella decora. J. Biol. Chem. 1990, 265, 10143-10147.

11. Mazur, P.; Henzel, W.J.; Seymour, J.L.; Lazarus, R.A. Ornatins: potent glycoprotein IIb-IIIa antagonists and platelet aggregation inhibitors from the leech Placobdella ornata. Eur. J. Biochem. 1991, 202, 1073-1082.

12. Wang, X.; Coons, L.B.; Taylor, D.B.; Stevens, S.E., Jr.; Gartner, T.K. Variabilin, a novel RGD-containing antagonist of glycoprotein IIb-IIIa and platelet aggregation inhibitor from the hard tick Dermacentor variabilis. J. Biol. Chem. 1996, 271, 17785-17790.

13. Bjarnason, J.B.; Fox, J.W. Hemorrhagic metalloproteinases from snake venoms. Pharmacol. Ther. 1994, 62, 325-372.

14. Lu, X.; Lu, D.; Scully, M.F.; Kakkar, V.V. Snake venom metalloproteinase containing a disintegrin-like domain, its structure-activity relationships at interacting with integrins. Curr. Med. Chem. Cardiovasc. Hematol. Agents 2005, 3, 249-260.

15. Weskamp, G.; Blobel, C.P. A family of cellular proteins related to snake venom disintegrins. Proc. Natl. Acad. Sci. USA 1994, 91, 2748-2751.

16. Wolfsberg, T.G.; Primakoff, P.; Myles, D.G.; White, J.M. ADAM, a novel family of membrane proteins containing A Disintegrin and Metalloproteinase domain: multipotential functions in cell-cell and cell-matrix interactions. J. Cell Biol. 1995, 131, 275-278.

17. Wolfsberg, T.G.; Straight, P.D.; Gerena, R.L.; Huovila, A.P.; Primakoff, P.; Myles, D.G.; White, J.M. ADAM, a widely distributed and developmentally regulated gene family encoding membrane proteins with a disintegrin and metalloproteinase domain. Dev. Biol. 1995, 169, 378-383.

18. Wolfsberg, T.G.; White, J.M. ADAMs in fertilization and development. Dev. Biol. 1996, 180, 389-401.

19. Kuno, K.; Kanada, N.; Nakashima, E.; Fujiki, F.; Ichimura, F.; Matsushima, K. Molecular cloning of a gene encoding a new type of metalloproteinase-disintegrin family protein with thrombospondin motifs as an inflammation associated gene. J. Biol. Chem. 1997, 272, 556-562.

20. Tang, B.L.; Hong, W. ADAMTS: a novel family of proteases with an ADAM protease domain and thrombospondin 1 repeats. FEBS Lett. 1999, 445, 223-225.

21. Hirohata, S.; Wang, L.W.; Miyagi, M.; Yan, L.; Seldin, M.F.; Keene, D.R.; Crabb, J.W.; Apte, S.S. Punctin, a novel ADAMTS-like molecule, ADAMTSL-1, in extracellular matrix. J. Biol. Chem. 2002, 277, 12182-12189.

22. Perry, A.C.; Jones, R.; Hall, L. Analysis of transcripts encoding novel members of the mammalian metalloproteinase-like, disintegrin-like, cysteine-rich (MDC) protein family and 
their expression in reproductive and non-reproductive monkey tissues. Biochem. J. 1995, 312, 239-244.

23. Selistre de Araujo, H.S.; de Souza, D.H.; Ownby, C.L. Analysis of a cDNA sequence encoding a novel member of the snake venom metalloproteinase, disintegrin-like, cysteine-rich (MDC) protein family from Agkistrodon contortrix laticinctus. Biochim. Biophys. Acta 1997, 1342, 109-115.

24. Lum, L.; Reid, M.S.; Blobel, C.P. Intracellular maturation of the mouse metalloproteinase disintegrin MDC15. J. Biol. Chem. 1998, 273, 26236-26247.

25. Herren, B.; Raines, E.; Ross, R. Expression of a disintegrin-like protein in cultured human vascular cells and in vivo. FASEB J. 1997, 11, 173-180.

26. Kratzschmar, J.; Lum, L.; Blobel, C.P. Metargidin, a membrane-anchored metalloproteinasedisintegrin protein with an RGD integrin binding sequence. J. Biol. Chem. 1996, 271, $4593-4596$.

27. Kärkkäinen, I.; Karhu, R.; Huovila, A.P. Assignment of the ADAM15 gene to human chromosome band 1q21.3 by in situ hybridization. Cytogenet. Cell Genet. 2000, 88, 206-207.

28. Kleino, I.; Ortiz, R.M.; Huovila, A.P. ADAM15 gene structure and differential alternative exon use in human tissues. BMC Mol. Biol. 2007, 8, 90-119.

29. Kleino, I.; Ortiz, R.M.; Yritys, M.; Huovila, A.P.; Saksela, K. Alternative splicing of ADAM15 regulates its interactions with cellular SH3 proteins. Cell Biochem. 2009, 108, 877-885.

30. Hurskainen, T.L.; Hirohata, S.; Seldin, M.F.; Apte, S.S. ADAM-TS5, ADAM-TS6, and ADAM-TS7, novel members of a new family of zinc metalloproteinases. General features and genomic distribution of the ADAM-TS family. J. Biol. Chem. 1999, 274, 25555-25563.

31. Becker, J.W.; Marcy, A.I.; Rokosz, L.L.; Axel, M.G.; Burbaum, J.J.; Fitzgerald, P.M.; Cameron, P.M.; Esser, C.K.; Hagmann, W.K.; Hermes, J.D.; Springer, J.P. Stromelysin-1: three-dimensional structure of the inhibited catalytic domain and of the C-truncated proenzyme. Protein Sci. 1995, 4, 1966-1976.

32. Kang, T.; Zhao, Y.G.; Pei, D.; Sucic, J.F.; Sang, Q.X. Intracellular activation of human adamalysin 19/disintegrin and metalloproteinase 19 by furin occurs via one of the two consecutive recognition sites. J. Biol. Chem. 2002, 277, 25583-25592.

33. Anders, A.; Gilbert, S.; Garten, W.; Postina, R.; Fahrenholz, F. Regulation of the alpha-secretase ADAM10 by its prodomain and proprotein convertases. FASEB J. 2001, 15, 1837-1879.

34. Fox, J.W.; Bjarnason, J.B. Zinc Metalloproteinases in Health and Disease; Hooper, N.M., Ed., Taylor \& Francis: Bristol, UK, 1996; pp. 47-81.

35. Marcinkiewicz, C. Functional characteristic of snake venom disintegrins: potential therapeutic implication. Curr. Pharm. Des. 2005, 11, 815-827.

36. Loechel, F.; Gilpin, B.J.; Engvall, E.; Albrechtsen, R.; Wewer, U.M. Human ADAM-12 (meltrin $\alpha$ ) is an active metalloproteinase. J. Biol. Chem. 1998, 273, 16993-16997.

37. Wolfsberg, T.G.; Bazan, J.F.; Blobel, C.P.; Myles, D.G.; Primakoff, P.; White, J.M. The precursor region of a protein active in sperm-egg fusion contains a metalloproteinase and a disintegrin domain: structural, functional, and evolutionary implications. Proc. Natl. Acad. Sci. USA 1993, 90, 10783-10787. 
38. Smith, K.M.; Gaultier, A.; Cousin, H.; Alfandari, D.; White, J.M.; DeSimone, D.W. The cysteine-rich domain regulates ADAM protease function in vivo. J. Cell Biol. 2002, 159, 893-902.

39. Zhong, J.L.; Poghosyan, Z.; Pennington, C.J.; Scott, X.; Handsley, M.M.; Warn, A.; Gavrilovic, J.; Honert, K.; Krüger, A.; Span, P.N.; et al. Distinct Functions of Natural ADAM-15 Cytoplasmic Domain Variants in Human Mammary Carcinoma. Mol. Cancer Res. 2008, 6, 383-394.

40. White, J.M.; Bridges, L.C.; DeSimone, D.W.; Tomczuk, M.; Wolfsberg, T.G. Introduction to the ADAM family. In The ADAM Family of Proteases: Proteases in Biology and Disease; Hooper N.M., Lendeckel, V.D., Eds.; Springer: Dordrecht, The Netherlands, 2005; Volume 4, pp. 1-28.

41. Langer, H.; May, A.E.; Bültmann, A.; Gawaz, M. ADAM 15 is an adhesion receptor for platelet GPIIb-IIIa and induces platelet activation. Thromb. Haemost. 2005, 94, 555-561.

42. Eto, K.; Huet, C.; Tarui, T.; Kupriyanov, S.; Liu, H.Z.; Puzon-McLaughlin, W.; Zhang, X.P.; Sheppard, D.; Engvall, E.; Takada, Y. Functional classification of ADAMs based on a conserved motif for binding to integrin alpha 9beta 1: implications for sperm-egg binding and other cell interactions. J. Biol. Chem. 2002, 277, 17804-17810.

43. Tomczuk, M.; Takahashi, Y.; Huang, J.; Murase, S.; Mistretta, M.; Klaffky, E.; Sutherland, A.; Bolling, L.; Coonrod, S.; Marcinkiewicz, C.; et al. Role of multiple betal integrins in cell adhesion to the disintegrin domains of ADAMs 2 and 3. Exp. Cell Res. 2003, 290, 68-81.

44. Lu, X.; Lu, D.; Scully, M.; Kakkar, V. ADAM proteins - therapeutic potential in cancer. Curr. Cancer Drug Targets 2008, 8, 720-32.

45. Adler, M.; Lazarus, R.A.; Dennis, M.S.; Wagner, G. Solution structure of kistrin, a potent platelet aggregation inhibitor and GP IIb-IIIa antagonist. Science 1991, 253, 445-448.

46. Adler, M.; Wagner, G. Sequential 1H NMR assignments of kistrin, a potent platelet aggregation inhibitor and glycoprotein IIb-IIIa antagonist. Biochemistry 1992, 31, 1031-1039.

47. Adler, M.; Carter, P.; Lazarus, R.A.; Wagner, G. Cysteine pairing in the glycoprotein IIb-IIIa antagonist kistrin using NMR, chemical analysis, and structure calculations. Biochemistry 1993, 32, 282-289.

48. Senn, H.; Klaus, W. The nuclear magnetic resonance solution structure of flavoridin, an antagonist of the platelet GP IIb-IIIa receptor. J. Mol. Biol. 1993, 232, 907-25.

49. Saudek, V.; Atkinson, R.A.; Lepage, P.; Pelton, J.T. The secondary structure of echistatin from 1H-NMR, circular-dichroism and Raman spectroscopy. Eur. J. Biochem. 1991, 202, 329-338.

50. Cooke, R.M.; Carter, B.G.; Martin, D.M.A.; Murray-Rust, P.; Weir, M.P. Nuclear magnetic resonance studies of the snake toxin echistatin. $1 \mathrm{H}$ resonance assignments and secondary structure. Eur. J. Biochem. 1991, 202, 323-328.

51. Cooke, R.M.; Carter, B.G.; Murray-Rust, P.; Hartshorn, M.J.; Herzyk, P.; Hubbard, R.E. The solution structure of echistatin: evidence for disulphide bond rearrangement in homologous snake toxins. Protein Eng. 1992, 5, 473-477.

52. Jaseja, M.; Smith, K.J.; Lu, X.; Williams, J.A.; Trayer, H.; Trayer, I.P.; Hyde, E.I. 1H-NMR studies and secondary structure of the RGD-containing snake toxin, albolabrin. Eur. J. Biochem. 1993, 218, 853-860. 
53. Jaseja, M.; Lu, X.; Williams, J.A.; Sutcliffe, M.J.; Kakkar, V.V.; Parslow, R.A.; Hyde, E.I. $1 \mathrm{H}-\mathrm{NMR}$ assignments and secondary structure of dendroaspin, an RGD-containing glycoprotein IIb-IIIa (alpha IIb-beta 3) antagonist with a neurotoxin fold. Eur. J. Biochem. 1994, 226, 861-868.

54. Fujii, Y.; Okuda, D.; Fujimoto, Z.; Horii, K.; Morita, T.; Mizuno, H. Crystal structure of trimestatin, a disintegrin containing a cell adhesion recognition motif RGD. J. Mol. Biol. 2003, 332, 1115-1122.

55. McLane, M.A.; Marcinkiewicz, C.; Vijay-Kumar, S.; Wierzbicka-Patynowski, I.; Niewiarowski, S. Viper venom disintegrins and related molecules. Proc. Soc. Exp. Biol. Med. 1998, 219, 109-119.

56. Lu, X.; Lu, D.; Scully, M.F.; Kakkar, V.V. Structure-activity relationship studies on ADAM protein-integrin interactions. Cardiovasc. Hematol. Agents Med. Chem. 2007, 5, 29-42.

57. Gomis-Ruth, F.X. Structural aspects of the metzincin clan of metalloendopeptidases. Mol. Biotechnol. 2003, 24, 157-202.

58. White, J.M. ADAMs: modulators of cell-cell and cell-matrix interactions. Curr. Opin. Cell Biol. 2003, 15, 598-606.

59. Terra, R.M.; Pinto, A.F.; Guimarães, J.A.; Fox, J.W. Proteomic profiling of snake venom metalloproteinases (SVMPs): insights into venom induced pathology. Toxicon 2009, 54, 836-844.

60. Baldo, C.; Tanjoni, I.; León, I.R.; Batista, I.F.; Della-Casa, M.S.; Clissa, P.B.; Weinlich, R.; Lopes-Ferreira, M.; Lebrun, I.; Amarante-Mendes, G.P.; et al. BnP1, a novel P-I metalloproteinase from Bothrops neuwiedi venom: biological effects benchmarking relatively to jararhagin, a P-III SVMP. Toxicon 2008, 51, 54-65.

61. Guan, H.H.; Goh, K.S.; Davamani, F.; Wu, P.L.; Huang, Y.W.; Jeyakanthan, J.; Wu, W.G.; Chen, C.J. Structures of two elapid snake venom metalloproteases with distinct activities highlight the disulfide patterns in the D domain of ADAMalysin family proteins. J. Struct. Biol. 2010, 169, 294-303.

62. Liu, H.; Shim, A.H.; He, X. Structural characterization of the ectodomain of a disintegrin and metalloproteinase-22 (ADAM22), a neural adhesion receptor instead of metalloproteinase: insights on ADAM function. J. Biol. Chem. 2009, 284, 29077-29086.

63. Takeda, S.; Igarashi, T.; Mori, H.; Araki, S. Crystal structures of VAP1 reveal ADAMs' MDC domain architecture and its unique C-shaped scaffold. EMBO J. 2006, 25, 2388-2396.

64. Igarashi, T.; Araki, S.; Mori, H.; Takeda, S. Crystal structures of catrocollastatin/VAP2B reveal a dynamic, modular architecture of ADAM/adamalysin/reprolysin family proteins. FEBS Lett. 2007, 581, 2416-2422.

65. Takeda, S. VAP1: snake venom homolog of mammalian ADAMs. In Handbook of Metalloproteins; Messerschmidt, A., Ed.; John Wiley \& Sons, Inc.: Somerset, NJ, USA, 2008; DOI: 10.1002/0470028637.met234.

66. Takeda, S. Three-dimensional domain architecture of the ADAM family proteinases. Semin. Cell Dev. Biol. 2009, 20, 146-152. 
67. Nath, D.; Slocombe, P.M.; Stephens, P.E.; Warn, A.; Hutchinson, G.R.; Yamada, K.M.; Docherty, A.J.; Murphy, G. Interaction of metargidin (ADAM-15) with alphavbeta3 and alpha5beta1 integrins on different haemopoietic cells. J. Cell Sci. 1999, 112, 579-587.

68. Eto, K.; Puzon-McLaughlin, W.; Sheppard, D.; Sehara-Fujisawa, A.; Zhang, X.P.; Takada, Y. RGD-independent binding of integrin alpha9beta1 to the ADAM-12 and -15 disintegrin domains mediates cell-cell interaction. J. Biol. Chem. 2000, 275, 34922-34930.

69. Fourie, A.M.; Coles, F.; Moreno, V.; Karlsson, L. Catalytic activity of ADAM8, ADAM15, and MDC-L (ADAM28) on synthetic peptide substrates and in ectodomain cleavage of CD23. J. Biol. Chem. 2003, 278, 30469-30477.

70. Seals, D.F.; Courtneidge, S.A. The ADAMs family of metalloproteinases: multidomain proteins with multiple functions. Genes Dev. 2003, 17, 7-30.

71. Taooka, Y.; Chen, J.; Yednock, T.; Sheppard, D. The integrin alpha9beta1 mediates adhesion to activated endothelial cells and transendothelial neutrophil migration through interaction with vascular cell adhesion molecule-1. J. Cell Biol. 1999, 145, 413-420.

72. Kanayama, M.; Kurotaki, D.; Morimoto, J.; Asano, T.; Matsui, Y.; Nakayama, Y.; Saito, Y.; Ito, K.; Kimura, C.; Iwasaki, N.; et al. Alpha9 integrin and Its Ligands Constitute Critical Joint Microenvironments for Development of Autoimmune Arthritis. J. Immunol. 2009, 182, $8015-8025$.

73. Lu, D.; Chung, K.F.; Xia, M.; Lu, X.; Scully, M.; Kakkar, V. Integrin binding characteristics of the disintegrin-like domain of ADAM-15. Thromb. Haemost. 2006, 96, 642-651.

74. Charrier, L.; Yan, Y.; Nguyen, H.T.; Dalmasso, G.; Laboisse, C.L.; Gewirtz, A.T.; Sitaraman, S.V.; Merlin, D. ADAM-15/metargidin mediates homotypic aggregation of human $\mathrm{T}$ lymphocytes and heterotypic interactions of T lymphocytes with intestinal epithelial cells. J. Biol. Chem. 2007, 282, 16948-16958.

75. Zhang, X.P.; Kamata, T.; Yokoyama, K.; Puzon-McLaughlin, W.; Takada, Y. Specific interaction of the recombinant disintegrin-like domain of MDC-15 (metargidin, ADAM-15) with integrin alphavbeta3. J. Biol. Chem. 1998, 273, 7345-7350.

76. Mosnier, J.F.; Jarry, A.; Bou-Hanna, C.; Denis, M.G.; Merlin, D.; Laboisse, C.L. ADAM15 upregulation and interaction with multiple binding partners in inflammatory bowel disease. Lab. Invest. 2006, 86, 1064-1073.

77. Eliceiri, B.P.; Cheresh, D.A. Role of alpha v integrins during angiogenesis. Cancer J. 2000, 3, S245-S249.

78. Kim, J.B.; Islam, S.; Kim, Y.J.; Prudoff, R.S.; Sass, K.M.; Wheelock, M.J.; Johnson, K.R. $\mathrm{N}-$ Cadherin extracellular repeat 4 mediates epithelial to mesenchymal transition and increased motility. J. Cell Biol. 2000, 151, 1193-1206.

79. Jeon, O.H.; Kim, D.; Choi, Y.J.; Kim, S.H.; Choi, W.S.; Kim, D.S. Novel function of human ADAM15 disintegrin-like domain and its derivatives in platelet aggregation. Thromb. Res. 2007, 119, 609-619.

80. Lu, D.; Xie, S.; Sukkar, M.B.; Lu, X.; Scully, M.F.; Chung, K.F. Inhibition of airway smooth muscle adhesion and migration by the disintegrin domain of ADAM-15. Am. J. Respir. Cell Mol. Biol. 2007, 37, 494-500. 
81. Pierschbacher, M.D.; Ruoslahti, E. Variants of the cell recognition site of fibronectin that retain attachment-promoting activity. Proc. Natl. Acad. Sci. USA 1984, 81, 5985-5988.

82. Mould, A.P.; Burrows, L.; Humphries, M.J. Identification of amino acid residues that form part of the ligand-binding pocket of integrin alpha5 beta1. J. Biol. Chem. 1998, 273, 25664-25672.

83. Murphy, G. The ADAMs: Signalling scissors in the tumour microenvironment. Nat. Rev. Cancer 2008, 8, 929-941.

84. Horiuchi, K,; Weskamp, G.; Lum, L.; Hammes, H.P.; Cai, H.; Brodie, T.A.; Ludwig, T.; Chiusaroli, R.; Baron, R.; Preissner, K.T.; et al. Potential role for ADAM15 in pathological neovascularization in mice. Mol. Cell Biol. 2003, 23, 5614-5624.

85. Yamada, D.; Ohuchida, K.; Mizumoto, K.; Ohhashi, S.; Yu, J.; Egami, T.; Fujita, H.; Nagai, E.; Tanaka, M. Increased expression of ADAM 9 and ADAM 15 mRNA in pancreatic cancer. Anticancer Res. 2007, 27, 793-799.

86. Najy, A.J.; Day, K.C.; Day, M.L. ADAM15 supports prostate cancer metastasis by modulating tumor cell-endothelial cell interaction. Cancer Res. 2008, 68, 1092-1099.

87. Najy, A.J.; Day, K.C.; Day, M.L. The ectodomain shedding of E-cadherin by ADAM15 supports ErbB receptor activation. J. Biol. Chem. 2008, 283, 18393-18401.

88. Ross, R. Atherosclerosis-an inflammatory disease. N. Engl. J. Med. 1999, 340, 115-126.

89. Herren, B.; Raines, E.W.; Ross, R. Expression of a disintegrin-like protein in cultured human vascular cells and in vivo. FASEB J. 1997, 11, 173-180.

90. Yuan, S.Y.; Ustinova, E.E.; Wu, M.H.; Tinsley, J.H.; Xu, W.; Korompai, F.L.; Taulman, A.C. Protein kinase $\mathrm{C}$ activation contributes to microvascular barrier dysfunction in the heart at early stages of diabetes. Circ. Res. 2000, 87, 412-417.

91. Matsuki, K.; Sasho, T.; Nakagawa, K.; Tahara, M.; Sugioka, K.; Ochiai, N.; Ogino, S.; Wada, Y.; Moriya, H. RGD peptide-induced cell death of chondrocytes and synovial cells. J. Orthop. Sci. 2008, 13, 524-532.

92. Zhou, Q.; Nakada, M.T.; Arnold, C.; Shieh, K.Y.; Markland, F.S., Jr. Contortrostatin, a dimeric disintegrin from Agkistrodon contortrix contortrix, inhibits angiogenesis. Angiogenesis 1999, 3, 259-269.

93. Wu, W.B.; Peng, H.C.; Huang, T.F. Disintegrin causes proteolysis of beta-catenin and apoptosis of endothelial cells. Involvement of cellcell and cell-ECM interactions in regulating cell viability. Exp. Cell Res. 2003, 286, 115-127.

94. Chung, K.H.; Kim, S.H.; Han, K.Y.; Sohn, Y.D.; Chang, S.I.; Baek, K.H.; Jang, Y.; Kim, D.S.; Kang, I.C. Inhibitory effect of salmosin, a Korean snake venom-derived disintegrin, on the integrin alphav-mediated proliferation of SK-Mel-2 human melanoma cells. J. Pharm. Pharmacol. 2003, 55, 1577-1582.

95. Kerr, J.S.; Slee, A.M.; Mousa, S.A. The alpha v integrin antagonists as novel anticancer agents: an update. Expert Opin. Investig. Drugs 2002, 11, 1765-1774.

96. Hallahan, D.E.; Qu, S.; Geng, L.; Cmelak, A.; Chakravarthy, A.; Martin, W.; Scarfone, C.; Giorgio, T. Radiation-mediated control of drug delivery. Am. J. Clin. Oncol. 2001, 24, 473-480.

97. Coller, B.S. Anti-GPIIb/IIIa drugs: current strategies and future directions. Thromb. Haemost. 2001, 86, 427-443. 
98. Xiao, T.; Takagi, J.; Coller, B.S.; Wang, J.H.; Springer, T.A. Structural basis for allostery in integrins and binding to fibrinogen-mimetic therapeutics. Nature 2004, 432, 59-67.

99. Pinto, A.F.; Terra, R.M.; Guimaraes, J.A.; Fox, J.W. Mapping von Willebrand factor A domain binding sites on a snake venom metalloproteinase cysteinerich domain. Arch. Biochem. Biophys. 2007, 457, 41-46.

100. Cal, S.; Freije, J.M.; López, J.M.; Takada, Y.; López-Otín, C. ADAM 23/MDC3, a human disintegrin that promotes cell adhesion via interaction with the alphavbeta3 integrin through an RGD-independent mechanism. Mol. Biol. Cell 2000, 11, 1457-1469.

101. Bridges, L.C.; Tani, P.H.; Hanson, K.R.; Roberts, C.M.; Judkins, M.B.; Bowditch, R.D. The lymphocyte metalloprotease MDC-L (ADAM 28) is a ligand for the integrin $\alpha_{4} \beta_{1}$. J. Biol. Chem. 2002, 277, 3784-3792.

(C) 2010 by the authors; licensee MDPI, Basel, Switzerland. This article is an open access article distributed under the terms and conditions of the Creative Commons Attribution license (http://creativecommons.org/licenses/by/3.0/). 